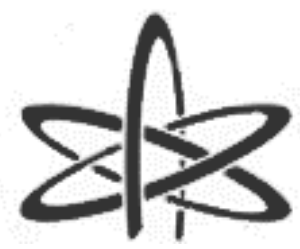

BJRS
BRAZILIAN JOURNAL

$\mathrm{OF}$

RADIATION SCIENCES

09-01 (2021) 01-14

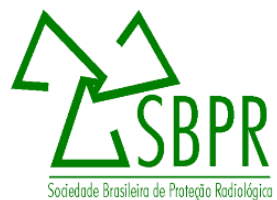

\title{
On the spectrum of discrete-ordinates neutron transport problems
}

\author{
Curbelo ${ }^{\mathrm{a}}$ J.P. \\ ${ }^{a}$ Universidade Estadual de Santa Cruz, Departamento de Ciências Exatas e Tecnológicas, Campus Soane Nazaré de \\ Andrade, Rodovia Jorge Amado, Km 16, Bairro Salobrinho CEP 45662-900, Ilhéus, BA, Brasil \\ jpcurbelo@uesc.br
}

\begin{abstract}
Over the last six decades, the discrete spectrum of the neutron transport operator has been widely studied. Significant theoretical results can be found in the literature regarding the one-speed linear transport equation with anisotropic scattering. In this study, the discrete-ordinates $\left(S_{N}\right)$ transport problem with anisotropic scattering has been considered and the discrete spectrum results in multiplying media have been corroborated. The numerical results obtained for the dominant $S_{N}$ eigenvalues agreed with the ones for the analytic problem reported in the literature up to a triplet scattering order. A compact methodology to perform the spectral analysis to multigroup $S_{N}$ problems with high anisotropy order in the scattering and fission reactions is also presented in this paper.
\end{abstract}

Keywords: transport equation, discrete ordinates, spectral analysis, discrete eigenvalues, anisotropic scattering 


\section{|1. INTRODUCTION}

Case (1960) presented a general procedure with the intent to analytically solve the Boltzmann Transport Equation (BTE) by the expansion of its solution into a complete set of eigenfunctions [1]. This is, undoubtedly, one of the most remarkable studies in the field of neutral particle transport theory [2]. Even though eigenfunctions might present a lack of utility in the solution to practical nuclear engineering problems, this method has been applied among different fields in physics seeking a comprehensive mathematical understanding [2,3]. Case found an analytic solution to the steadystate, homogeneous BTE in slab geometry considering one-speed particles and isotropic scattering. Over the past 60 years, the method has been applied to more complex problems that may consider energy dependence, multiplying media, anisotropic scattering, heterogeneity, and/or multiple dimensions [2, 4].

Case proved that the solutions are given by two discrete modes corresponding to a \pm pair of eigenvalues that lie outside interval $(-1 ;+1)$, in addition to a complimentary continuous eigenvalue spectrum over the interval $[-1 ;+1][1]$. This pair of discrete dominant eigenvalues are conventionally referred to as $c$-eigenvalues [5] since they depend on the material cross section by

$$
c=\frac{\Sigma_{S}+v \Sigma_{f}}{\Sigma_{T}} .
$$

In regard to obtaining a discrete eigenvalue spectrum, besides the monoenergetic problem with isotropic scattering [1], the main results have been applied to problems considering linearly anisotropic scattering [6], and more recently, arbitrary-order anisotropic scattering [4, 7, 8]. In Section 2 of this paper, the methodology described by Sahni and Tureci [4] is summarized and the main results for all the mentioned cases are presented.

To the best of this author's knowledge, no published work has extended these results to include multigroup these results to multigroup transport problems in the discrete-ordinates $\left(S_{N}\right)$ formulation considering arbitrary order of anisotropy on the scattering and fission reactions. The procedures described here present a general solution to the problem cited before, including the possibility of obtaining eigenvalues over the complex plane. The spectral analysis for the $S_{N}$ BTE that supports the discrete eigenvalues of the Case's spectrum has also been performed. 


\section{MATERIALS AND METHODS}

According to the notation used by Sahni and Tureci [4], the steady-state BTE for one-speed neutrons in a slab-geometry homogeneous media can be written as

$$
\mu \frac{\partial}{\partial x} \psi(x, \mu)+\Sigma_{T} \psi(x, \mu)=\frac{c \Sigma_{T}}{2} \sum_{l=0}^{L} b_{l} P_{l}(\mu) \int_{-1}^{1} d \mu^{\prime} P_{l}\left(\mu^{\prime}\right) \psi\left(x, \mu^{\prime}\right)
$$

In Equation (1), the conventional terms apply: $\Sigma_{T}$ is the total macroscopic cross-section, $\mathrm{c}$ is the mean number of secondary neutrons per collision, $b_{l}$ depend on the scattering function with $b_{0}=1$, and $P_{l}(\mu)$ are the Legendre polynomials of degree $l$. The quantities $x$ and $\mu \in[-1 ;+1]$ are the spatial coordinate and the direction variable, respectively, and $\psi(x, \mu)$ is the neutron flux.

To solve Equation (1), the method of separation of variables is applied by the substitution

that yields

$$
\psi(x, \mu)=\phi_{\xi}(\mu) e^{\frac{-\Sigma_{T} x}{\xi}}
$$

$$
\frac{1}{\mu} \phi_{\xi}(\mu)-\frac{c}{2 \mu} \sum_{l=0}^{L} b_{l} P_{l}(\mu) \int_{-1}^{1} d \mu^{\prime} P_{l}\left(\mu^{\prime}\right) \phi_{\xi}\left(\mu^{\prime}\right)=\frac{1}{\xi} \phi_{\xi}(\mu)
$$

At this point, the recursion relations for Legendre polynomials are applied along with some algebraic manipulations to obtain the transcendental equation

$$
\frac{c \xi}{2} \sum_{l=0}^{L} b_{l} \int_{-1}^{1} d \mu^{\prime} P_{l}\left(\mu^{\prime}\right) \phi_{\xi}\left(\mu^{\prime}\right) \int_{-1}^{1} d \mu \frac{P_{l}(\mu)}{\xi-\mu}-1=0 \quad, \quad \xi \notin[-1 ; 1]
$$

whose roots are the discrete values of $\xi$ and appear in \pm pairs. Moreover, if $\xi$ is a complex number, then its complex conjugate $\bar{\xi}$ is also a root.

From the material properties of the media (i.e., values of $c$ and $b_{l}$ ), one can deduct the type of the roots of Equation (4). Table 1 summarizes results for different anisotropy orders: isotropic scattering $(L=0)$ [1], linearly anisotropic scattering $(L=1)$ [6], and quadratic $(L=2)$ and triplet $(L=3)$ anisotropic scattering [4]. 
Table 1: Discrete eigenvalues under different conditions.

\begin{tabular}{|c|c|c|c|}
\hline & Conditions & Discre & eigenvalues \\
\hline \multirow{2}{*}{$L=0$} & $c>1$ & \multicolumn{2}{|c|}{ one pair of real roots $\pm \xi_{0}$} \\
\hline & $c<1$ & \multicolumn{2}{|l|}{ one pair of imaginary roots $\pm \xi_{0}$} \\
\hline \multirow{5}{*}{$L=1$} & $c>1$ & \multicolumn{2}{|l|}{ one pair of real roots $\pm \xi_{0}$} \\
\hline & $c<1, b_{1}<0$ & \multicolumn{2}{|l|}{ one pair of imaginary roots $\pm \xi_{0}$} \\
\hline & $b_{1}>0,1<c<1+1 / b_{1}$ & \multicolumn{2}{|l|}{ one pair of imaginary roots $\pm \xi_{0}$} \\
\hline & \multirow[b]{2}{*}{$b_{1}>0, c>1+1 / b_{1}$} & \multirow[b]{2}{*}{ one quartet or two pairs of roots } & $b_{1} c<3$ one real and one imaginary \\
\hline & & & $b_{1} c>3$ both imaginary or both real \\
\hline \multirow{5}{*}{$L=2$} & $c>1$ & \multicolumn{2}{|l|}{ one pair of real roots $\pm \xi_{0}$} \\
\hline & $c<1, b_{2}<0$ & \multicolumn{2}{|l|}{ one pair of imaginary roots $\pm \xi_{0}$} \\
\hline & $b_{2}>0,1<c<2 / 3\left(1+1 / b_{2}\right)$ & \multicolumn{2}{|l|}{ one pair of imaginary roots $\pm \xi_{0}$} \\
\hline & \multirow[b]{2}{*}{$b_{2}>0, c>2 / 3\left(1+1 / b_{2}\right)$} & \multirow[b]{2}{*}{ one quartet or two pairs of roots } & $b_{2} c<5$ one real and one imaginary \\
\hline & & & $b_{2} c>5$ both imaginary or both real \\
\hline \multirow{4}{*}{$L=3$} & $c>1$ & \multicolumn{2}{|l|}{ one pair of real roots $\pm \xi_{0}$} \\
\hline & $c<1, b_{3}<0$ & \multicolumn{2}{|l|}{ one pair of imaginary roots $\pm \xi_{0}$} \\
\hline & $b_{3}>0,1<c<6 / 11\left(1+1 / b_{3}\right)$ & \multicolumn{2}{|l|}{ one pair of imaginary roots $\pm \xi_{0}$} \\
\hline & $b_{3}>0, c>6 / 11\left(1+1 / b_{3}\right)$ & one quartet or two pairs of roots & $\begin{array}{ll}b_{3} c<7 & \text { one real and one imaginary } \\
b_{3} c>7 & \text { both imaginary or both real }\end{array}$ \\
\hline
\end{tabular}


Source: Data from the work by Sahni and Tureci [4]. 


\subsection{Spectral analysis of the $S_{N}$ transport equations}

The time-independent multigroup $S_{N}$ BTE with $L-$ 'th order of anisotropy on both the scattering and fission terms within a region $Y$ of a multiplying slab $[9,10]$ is considered with appropriate boundary conditions

$$
\begin{gathered}
\mu_{m} \frac{d}{d x} \psi_{m g}(x)+\Sigma_{T g} \psi_{m g}(x)=\sum_{l=0}^{L} \frac{2 l+1}{2} P_{l}\left(\mu_{m}\right) \sum_{g^{\prime}=1}^{G} \Sigma_{S g^{\prime} \rightarrow g}^{(l)} \sum_{n=1}^{N} P_{l}\left(\mu_{n}\right) \omega_{n} \psi_{n g^{\prime}}(x) \\
+\frac{\chi_{g}}{k_{\text {eff }}} \sum_{l=0}^{L} \frac{2 l+1}{2} P_{l}\left(\mu_{m}\right) \sum_{g^{\prime}=1}^{G} v_{g^{\prime}} \Sigma_{f g^{\prime}} \sum_{n=1}^{N} P_{l}\left(\mu_{n}\right) \omega_{n} \psi_{n g^{\prime}}(x), \\
x \in Y, \quad m=1: N, \quad g=1: G .
\end{gathered}
$$

The angular quadrature of order $N$ is defined by the discrete directions $\left(\mu_{m}\right)$ and their associated weights $\left(\omega_{m}\right)$. The quantity $\psi_{m g}(x)$ is the angular flux of particles with energy within the group $g$ traveling in direction $\mu_{m} ; \chi_{g}$ represents the spectrum of neutrons appearing in group $g$ due to fission; $\Sigma_{S g^{\prime} \rightarrow g}^{(l)}$ and $\Sigma_{f g^{\prime}}^{(l)}$ are the Legendre moments of the macroscopic differential scattering and fission cross section, respectively; $v_{g^{\prime}}$ is the average number of neutrons in group $g^{\prime}$ released in each fission reaction; and $k_{e f f}$ is the multiplication factor.

Equation (5) can be rewritten aiming an analogous form to Equation (1) for the monoenergetic analytic problem as

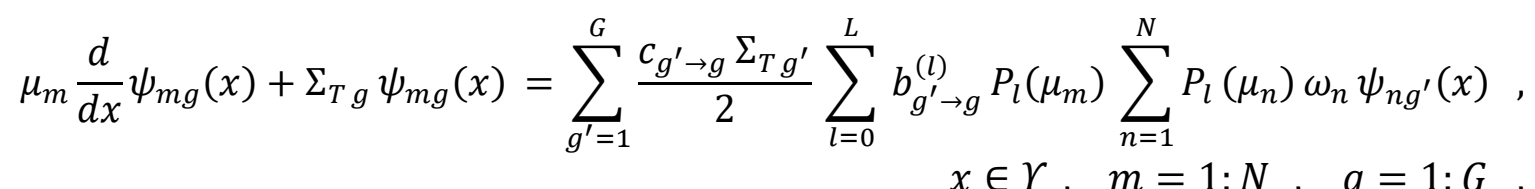

with the definitions:

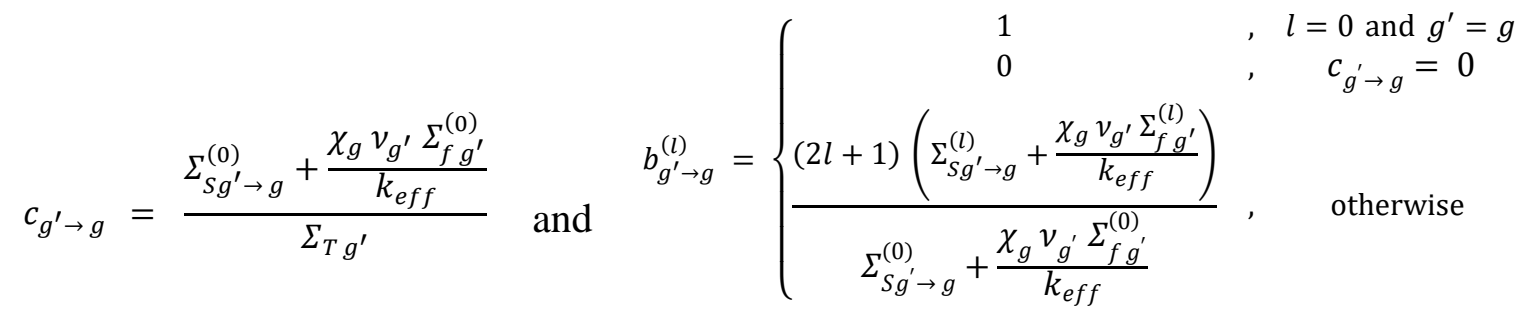

To solve the homogeneous equation, Equation (6), it is considered the function 


$$
\psi_{m g}(x)=a_{m g}(\xi) e^{-\frac{x}{\xi}}
$$

It is noteworthy that Equation (8) is analogous to the ansatz in Equation (2). Now, this expression is substituted into Equation (6) to obtain, after some operations, an eigenvalue problem of order $N G$

$$
\sum_{g^{\prime}=1}^{G} \sum_{n=1}^{N}\left\{\delta_{m, n} \delta_{g, g^{\prime}} \frac{\Sigma_{T g}}{\mu_{m}}-\frac{c_{g^{\prime} \rightarrow g} \Sigma_{T g^{\prime}} \omega_{n}}{2 \mu_{m}} \sum_{l=0}^{L} b_{g^{\prime} \rightarrow g}^{(l)} P_{l}\left(\mu_{m}\right) P_{l}\left(\mu_{n}\right)\right\} a_{n g^{\prime}}(\xi)=\frac{1}{\xi} a_{m g}(\xi)
$$

By solving this eigenvalue problem, a set of $N G$ linearly independent eigenfunctions defined in Equation (8) for $x \in Y$ is obtained.

\section{RESULTS AND DISCUSSION}

For high quadrature orders, the dominant eigenvalues obtained from the $S_{N}$ problem should agree with the discrete eigenvalues from the one-speed analytic problem. Sahni and Tureci [4] reported the discrete eigenvalues calculated considering several combinations of values of $c$ and $b_{l}$ for three test cases: (a) linearly anisotropic scattering $\left(b_{0}, b_{1} \neq 0\right.$, otherwise $\left.b_{l}=0\right)$, (b) isotropic +pure quadratic scattering $\left(b_{0}, b_{2} \neq 0\right.$, otherwise $\left.b_{l}=0\right)$, and (c) isotropic+pure triplet scattering $\left(b_{0}, b_{3} \neq 0\right.$, otherwise $\left.b_{l}=0\right)$.

The eigenvalue problem from the $S_{N}$ BTE, Equation (9), was solved for all the examples reported by Sahni and Tureci [4]. It was started at low orders of the Gauss-Legendre quadrature that were increased up to obtain $S_{N}$ results in agreement with the discrete Case's spectrum within a range of less than $100 \mathrm{pcm}$. The RMatrixEVD subroutine from the ALGLIB library [11] was used, in order to find the eigenvalues (real and imaginary parts) and eigenvectors of a general matrix.

Tables 2 to 4 show the results obtained from solving the $S_{N}$ eigenvalue problem published by Sahni and Tureci [4]. In all cases, the moduli of the dominant eigenvalues and the relative deviations in pcm with respect to the reference values are presented. One can observe that for a quadrature order $N=64$, the relative deviation is less than $80 \mathrm{pcm}$ in all cases. 
Table 2: The discrete eigenvalues for linearly anisotropic scattering $(L=1)^{a}$.

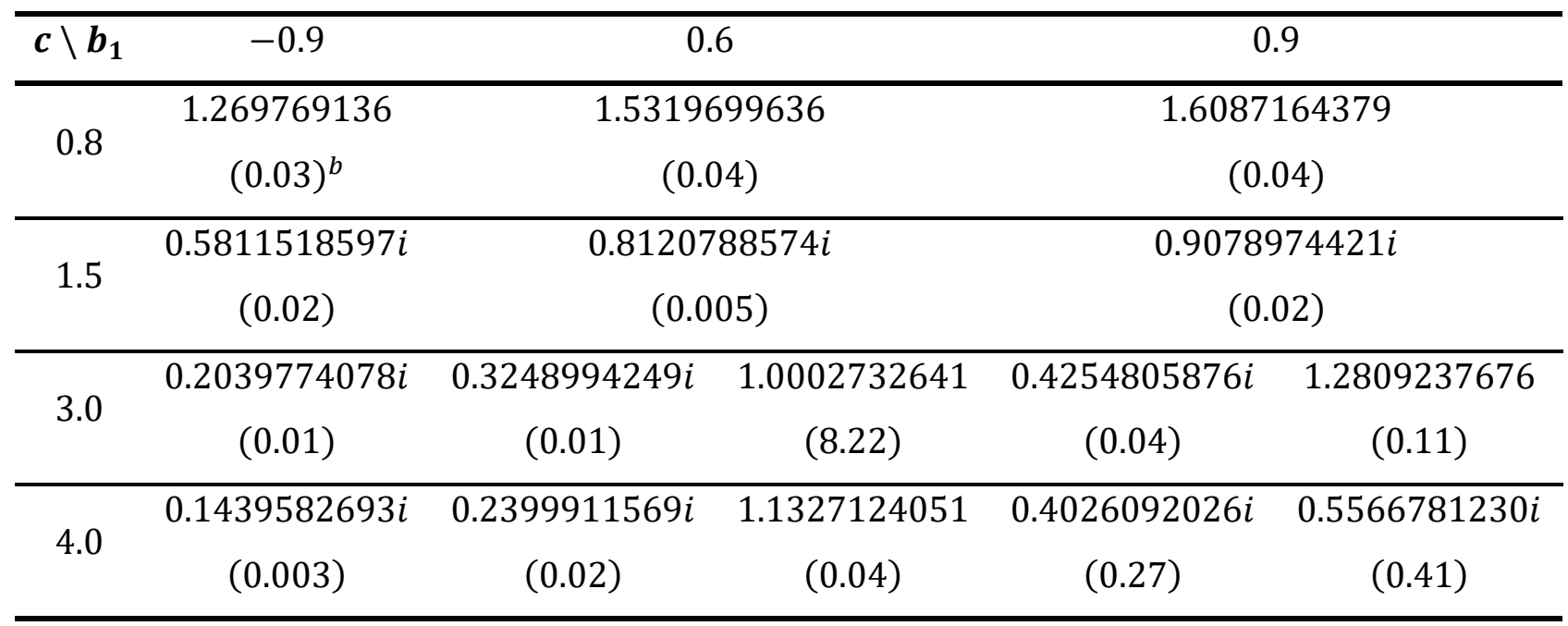

${ }^{a}$ Only the magnitude of real or purely imaginary eigenvalue pair is tabulated.

${ }^{b}$ relative deviation ( $p c m$ ) with respect to the discrete analytic eigenvalue [4].

Table 3: The discrete eigenvalues for linearly anisotropic scattering $(L=2)^{a}$.

\begin{tabular}{|c|c|c|c|c|c|}
\hline$c \backslash b_{2}$ & -1.0 & \multicolumn{2}{|c|}{0.5} & \multicolumn{2}{|c|}{1.5} \\
\hline \multirow{2}{*}{0.8} & 1.3902078564 & \multicolumn{2}{|c|}{1.4187852618} & \multicolumn{2}{|c|}{1.4486484525} \\
\hline & $(0.03)^{b}$ & \multicolumn{2}{|c|}{$(0.04)$} & \multicolumn{2}{|c|}{$(0.04)$} \\
\hline \multirow{2}{*}{1.5} & $0.712095766 i$ & \multirow{2}{*}{\multicolumn{2}{|c|}{$\begin{array}{c}0.67386471 i \\
(0.01)\end{array}$}} & $0.6317760051 i$ & 1.0309375337 \\
\hline & $(0.02)$ & & & $(0.004)$ & $(0.004)$ \\
\hline \multirow{2}{*}{3.0} & $0.2785204395 i$ & $0.2364960112 i$ & 1.0057660256 & $0.2045765429 i$ & 2.3007655785 \\
\hline & $(0.005)$ & $(0.0001)$ & $(0.01)$ & $(0.003)$ & (0.19) \\
\hline \multirow{2}{*}{4.0} & $0.2041791731 i$ & $0.1664595861 i$ & 1.0410302996 & $0.1415999511 i$ & $1: 6038876543 i$ \\
\hline & $(0.003)$ & $(0.003)$ & $(0.001)$ & $(0.01)$ & $(0.15)$ \\
\hline
\end{tabular}

${ }^{a}$ Only the magnitude of real or purely imaginary eigenvalue pair is tabulated.

${ }^{b}$ relative deviation $(p c m)$ with respect to the discrete analytic eigenvalue [4]. 
Table 4: The discrete eigenvalues for linearly anisotropic scattering $(L=3)^{a}$.

\begin{tabular}{|c|c|c|c|c|c|}
\hline$c \backslash b_{3}$ & -1.0 & \multicolumn{2}{|c|}{0.5} & \multicolumn{2}{|c|}{1.0} \\
\hline \multirow{2}{*}{0.8} & 1.4053567858 & \multicolumn{2}{|c|}{14090532489} & \multicolumn{2}{|c|}{1.4107235544} \\
\hline & $(0.03)^{b}$ & \multicolumn{2}{|c|}{$(0.03)$} & \multicolumn{2}{|c|}{$(0.03)$} \\
\hline \multirow{2}{*}{1.5} & $0.6841100404 i$ & \multirow{2}{*}{\multicolumn{2}{|c|}{$\begin{array}{c}0.6920605429 i \\
(0.01)\end{array}$}} & $0.6953423810 i$ & 1.0029060860 \\
\hline & $(0.01)$ & & & $(0.01)$ & $(0.125)$ \\
\hline \multirow{2}{*}{3.0} & $0.2399919939 i$ & $0.585796957 i$ & 1.0068223847 & $0.2663092301 i$ & 1.0936625073 \\
\hline & $(0.002)$ & $(0.004)$ & $(0.01)$ & $(0.01)$ & $(0.001)$ \\
\hline \multirow{2}{*}{10.0} & $0.0595745983 i$ & $00713391099 i$ & 1.4377274882 & $0.0778750654 i$ & $19751716742 i$ \\
\hline & $(80.2)$ & $(26.0)$ & $(0.03)$ & (13.0) & $(0.03)$ \\
\hline
\end{tabular}

${ }^{a}$ Only the magnitude of real or purely imaginary eigenvalue pair is tabulated.

${ }^{b}$ relative deviation $(\mathrm{pcm})$ with respect to the discrete analytic eigenvalue [4].

\subsection{Solution to the $S_{N}$ BTE}

As a result of the previous analysis, in this subsection, a methodology to obtain the analytic solution to the slab-geometry multigroup $S_{N}$ BTE in multiplying media [12-14] is proposed. It is remarked that the presented procedures can also be used to derive the homogeneous component of the general solution in fixed-source problems [15-20]. Equation (5) can be represented in matrix form as

$$
\frac{d}{d x} \boldsymbol{\Psi}=\mathbf{M} \boldsymbol{\Psi}
$$

where $\mathbf{M}$ is the $N G$-order square matrix with entries

$$
\begin{gathered}
M_{m g, n g^{\prime}}=\frac{1}{\mu_{m}}\left\{-\delta_{m, n} \delta_{g, g^{\prime}} \Sigma_{T g}+\sum_{l=0}^{L} \frac{2 l+1}{2} P_{l}\left(\mu_{m}\right) P_{l}\left(\mu_{n}\right) \omega_{n}\left[\Sigma_{s g^{\prime} \rightarrow g}^{(l)}+\frac{\chi_{g}}{k_{e f f}} v_{g^{\prime}} \Sigma_{f g^{\prime}}^{(l)}\right]\right\} \\
m=1: N, g=1: G, n=1: N, g^{\prime}=1: G
\end{gathered}
$$

and $\boldsymbol{\Psi}$ is a column matrix whose entries are $\psi_{m g}(x)$. The solution to the homogeneous system of ordinary differential equations in Equation (10) can be written as 


$$
\boldsymbol{\Psi}^{\mathrm{H}}(x)=\sum_{k=1}^{N G} \alpha_{k} v_{k} e^{\xi_{k} x}
$$

where $v_{k}$ are the eigenvectors associated to the eigenvalues $\xi_{k}$ of matrix $M$. Depending on the material parameters, the $N G$ eigenvalues $\xi_{k}$ can appear in \pm real pairs, imaginary or complex conjugate. Since the input of matrix $M$ are real numbers, the real eigenvalues will be associated to real eigenvectors, and the complex conjugate eigenvalues will be associated to complex conjugate eigenvectors.

As it is the case, when $\xi=p+q i$ and $\bar{\xi}=p-q i$ are a pair of eigenvalues, the eigenvectors associated to $\xi$ and $\bar{\xi}$ are $\mathbf{v}=\mathbf{a}+\mathbf{b} i$ and $\overline{\mathbf{v}}=\mathbf{a}-\mathbf{b} i$, respectively [21]. After some operations, two real-valued solutions are obtained

$$
\boldsymbol{\Psi}_{1}(x)=(a \cos q x-b \sin q x) e^{p x} \quad \text { and } \quad \Psi_{2}=(b \cos q x+a \sin q x) e^{p x} .
$$

Therefore, if $K_{R}$ real eigenvalues and $K_{C}$ complex conjugate pairs are found, i.e., $K_{R}+2 K_{C}=N G$, a set of $N G$ linearly independent eigenfunctions is obtained, and the solution to Equation (5) within region $Y$ is

$$
\begin{aligned}
\boldsymbol{\Psi}^{\mathrm{H}}(x)= & \sum_{k=1}^{K_{R}} \alpha_{k} \mathbf{v}_{k} e^{\xi_{k} x} \\
& \quad+\sum_{j=1}^{K_{C}}\left\{\beta_{j}\left(\mathbf{a}_{j} \cos q_{j} x-\mathbf{b}_{j} \sin q_{j} x\right)+\beta_{j}^{\prime}\left(\mathbf{b}_{j} \cos q_{j} x+\mathbf{a}_{j} \sin q_{j} x\right)\right\} e^{p_{j} x}
\end{aligned}
$$

where $\alpha_{i}, \beta_{j}$ and $\beta_{j}^{\prime}$ are arbitrary constants to be determined. Equation (14) can be represented in matrix form as

$$
\boldsymbol{\Psi}(x)=\left[\mathbf{M}_{\mathbf{R}} \mathbf{I}_{e \cos }(x)+\mathbf{N}_{\mathbf{R}} \mathbf{I}_{e \sin }(x)\right] \boldsymbol{\alpha}
$$

with the definition of matrices

$$
\mathbf{M}_{\mathbf{R}}=\left[\begin{array}{cccccccc}
\mathrm{v}_{1,1} & \cdots & \mathrm{v}_{1, K_{R}} & \mathrm{a}_{1,1} & \mathrm{~b}_{1,1} & \cdots & \mathrm{a}_{1, K_{C}} & \mathrm{~b}_{1, K_{C}} \\
\vdots & \ddots & \vdots & \vdots & \vdots & \ddots & \vdots & \vdots \\
\mathrm{v}_{N G, 1} & \cdots & \mathrm{v}_{N G, K_{R}} & \mathrm{a}_{N G, 1} & \mathrm{~b}_{N G, 1} & \cdots & \mathrm{a}_{N G, K_{C}} & \mathrm{~b}_{N G, K_{C}}
\end{array}\right]
$$




$$
\begin{gathered}
\mathbf{N}_{\mathbf{R}}=\left[\begin{array}{cccccccc}
0 & \cdots & 0 & -\mathrm{b}_{1,1} & \mathrm{a}_{1,1} & \cdots & -\mathrm{b}_{1, K_{C}} & \mathrm{a}_{1, K_{C}} \\
\vdots & \ddots & \vdots & \vdots & \vdots & \ddots & \vdots & \vdots \\
0 & \cdots & 0 & -\mathrm{b}_{N G, 1} & \mathrm{~b}_{N G, 1} & \cdots & -\mathrm{b}_{N G, K_{C}} & \mathrm{a}_{N G, K_{C}}
\end{array}\right], \\
\mathbf{I}_{e \cos }(x)=\operatorname{diag}\left\{e^{\xi_{1} x}, \cdots, e^{\xi_{K_{R}} x}, e^{p_{1} x} \cos q_{1} x, e^{p_{1} x} \cos q_{1} x, \cdots, e^{p_{K_{C}} x} \cos q_{K_{C}} x, e^{p_{K_{C}} x} \cos q_{K_{C}} x\right\} \\
\mathbf{I}_{e \sin }(x)=\operatorname{diag}\left\{0, \cdots, 0, e^{p_{1} x} \sin q_{1} x, e^{p_{1} x} \sin q_{1} x, \cdots, e^{p_{K_{C}} x} \sin q_{K_{C}} x, e^{p_{K_{C}} x} \sin q_{K_{C}} x\right\}
\end{gathered}
$$

and

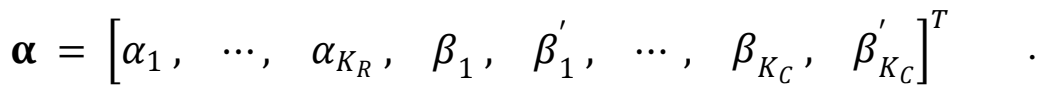

The terminology presented here for the spectral analysis can be used in the development and implementation of spectral nodal methods to obtain accurate and efficient numerical solutions to the $S_{N}$ BTE in slab-geometry. It is noteworthy that the notation used here is general and compact, nevertheless, it can be modified, and one should perform the construction of the matrices and the order of the operations aiming at computational efficiency.

\section{CONCLUSION}

In this paper, the spectral analysis of the $S_{N}$ BTE with anisotropic scattering has been performed and the results with the discrete Case's eigenvalues from the analytic transport problem have been compared. As one could anticipate, for a quadrature order high enough, the dominant $S_{N}$ eigenvalues agree with the discrete spectrum. A simplification for the procedures to obtain the analytic solution of the $S_{N}$ BTE considering high-order anisotropic events in the scattering and fission sources was also presented. This simplification has been presented in a compact form and includes the possibility of obtaining complex eigenvalues from the spectral analysis. The present methodology shall be applied not only to slab-geometry problems but also to multidimensional spectral nodal methods that use transverse integration procedures. 


\section{ACKNOWLEDGMENT}

This study was financed in part by the Coordenação de Aperfeiçoamento de Pessoal de Nível Superior - Brasil (CAPES) - Finance Code 001.

\section{REFERENCES}

[1] CASE, K. M. Elementary Solutions of the Transport Equation and Their Applications. Ann Phys, v. 9, p. 1-23, 1960.

[2] ZWEIFEL, P. F. Kenneth Case and his Singular "Eigenfunctions". Transport Theor Stat, v. 41, p. 406-417, 2012.

[3] MCCORMICK, N. J.; KUSCER, I. Singular eigenfunction expansions in neutron transport theory. In: LEWINS, J.; HENLEY, E. J. Advances in Nuclear Science and Technology, Academic Press, New York, USA, 1973. p. 181-282.

[4] SAHNI, D. C.; TURECI, R. G. Discrete Eigenvalues of Case Spectrum with Anisotropic Scattering. Nucl Sci Eng, v. 191, p. 121-135, 2018.

[5] MODAK, R. S.; SAHNI, D. C.; PARANJAPE, S. D. Evaluation of higher $k$-eigenvalues of the neutron transport equation by $S_{N}$-method. Ann Nucl Energy, v. 22, p. 359-366, 1995.

[6] MIKA, J. R. Neutron Transport with Anisotropic Scattering. Nucl Sci Eng, v. 11, p. 415-427, 1961.

[7] GARCIA, R. D. M.; SIEWERT, C. E. On Discrete Spectrum Calculations in Radiative Transfer. J Quant Spectrosc Ra, v. 42, p. 385-394, 1989.

[8] VAN DEN EYNDE, G. M.; BEAUWENS, R.; MUND, E. Calculating the Discrete Spectrum of the Transport Operator with Arbitrary Order Anisotropic Scattering. Transport Theor Stat, v. 36, p. 179-197, 2007.

[9] PRINJA, A.K.; LARSEN, E.W. General principles of neutron transport. In: CACUCI, D.G. Handbook of nuclear engineering, $1^{\text {st }}$ ed., New York, USA: Springer Science + Business Media, 2010. p.427-542. 
[10] MORATO, S.; BERNAL, A. ; Miró, R.; Roman, J. E. ; Verdú, G.; Calculation of $\lambda$ modes of the multi-group neutron transport equation using the discrete ordinates and Finite Difference Method. Ann Nucl Energy, v. 137, p. 107077, 2020.

[11] ALGLIB PROJECT, ALGLIB-numerical analysis library 1999-2021. Available at <www.alglib.net> Last accessed: 14 Dec. 2020.

[12] DE ABREU, M. P.; ALVES FILHO, H.; BARROS, R. C. A numerical method for multigroup slab-geometry eigenvalue problems in transport theory with no spatial truncation error Transport Theor Stat, v. 25, p. 61-83, 1996.

[13] BARROS, R. C.; ALVES FILHO, H.; ORELLANA E. T. V.; DA SILVA, F. C.; DO COUTO, N.; DOMINGUEZ, D. S.; HERNÁNDEZ, C. R. G. The application of spectral nodal methods to discrete ordinates and diffusion problems in Cartesian geometry for neutron multiplying system. Prog Nucl Energy, v. 42, p. 385-426, 2003.

[14] MORAES, L. R. C.; ALVES FILHO, H.; BARROS, R. C. Calculation of Neutron Interior Source Distribution Within Subcritical Fission-Chain Reacting Systems for a Prescribed Power Density Generation. In: INTERNATIONAL NUCLEAR ATLANTIC CONFERENCE, 2017, Belo Horizonte, Brazil, 2017. Associação Brasileira de Energia Nuclear (ABEN), 2017. P. 1-14.

[15] BARROS, R. C.; LARSEN, E. W. A numerical method for one-group slab-geometry discrete ordinates problems with no spatial truncation error. Nucl Sci Eng, v. 104, p. 199-208, 1990.

[16] DE ABREU, M. P. Numerical methods for the generation of the spectrum of the multigroup slab-geometry discrete ordinates operator in neutron transport theory. Ann Nucl Energy, v. 29, p. 1837-1853, 2002.

[17] SILVA , D. M.; LYDIA, E. J.; GUIDA, M. R.; ZANI, J. H.; ALVES FILHO, H.; BARROS, R. C. Analytical methods for computational modeling of fixed-source slab-geometry discrete ordinates transport problems: Response matrix and hybrid $S_{N}$. Prog Nucl Energy, v. 69, p. 77-84, 2013.

[18] MENEZES, W. A.; ALVES FILHO, H.; BARROS, R.C. Spectral Green's function nodal method for multigroup $S_{N}$ problems with anisotropic scattering in slab-geometry non-multiplying media. Ann Nucl Energy, v. 64, p. 270-275, 2014. 
[19] OlIVA, A. M.; ALVES FILHO, H.; SILVA , D. M.; GARCIA, C. R. The spectral nodal method applied to multigroup $S_{N}$ neutron transport problems in One-Dimensional geometry with Fixed-Source. Prog Nucl Energy, v. 105, p.106-113, 2018.

[20] CURBELO, J. P.; DA SILVA, O. P.; BARROS, R. C. An adjoint technique applied to slabgeometry source-detector problems using the generalized spectral Green's function nodal method. J Comput Theor Transp, v. 47, p. 278-299, 2018.

[21] EDWARDS, C. H.; PENNEY, D. E. Differential Equations \& Linear Algebra, $6^{\text {th }}$ ed. USA: Pearson, 2008. 